\title{
LncRNA MALAT1/MiR-145 Adjusts IL-1 $\beta$-Induced Chondrocytes Viability and Cartilage Matrix Degradation by Regulating ADAMTS5 in Human Osteoarthritis
}

\author{
Chengyao Liu' ${ }^{1}$ Shan Ren ${ }^{1}$, Shifeng Zhao², and Yandong Wang ${ }^{3}$ \\ Departments of ${ }^{1}$ Bone and Joint Surgery and ${ }^{2}$ Dermatology, The Sixth People's Hospital of Jinan City \\ (Zhangqiu People's Hospital affiliated to Jining Medical University), Shandong; \\ ${ }^{3}$ Department of Orthopedics, the Forth Hospital of Yulin (Xingyuan Hospital), West Yulin, Shaanxi, China.
}

Purpose: Accumulating evidence suggests that microRNA-145 (miR-145) plays an important role in osteoarthritis (OA), which is a chronic progressive joint disease. Long noncoding RNA metastasis-associated lung adenocarcinoma transcript 1 (MALAT1) promotes metastasis in cancers and functions as a sponge for miR-145. However, the role of MALAT1/miR-145 in OA pathogenesis has not yet been elucidated.

Materials and Methods: The expression of MALAT1 and miR-145 was examined by quantitative real-time PCR; the interaction between miR-145, MALAT1 and a disintegrin and metalloproteinase with thrombospondin motifs (ADAMTS) 5 was verified by luciferase reporter assay. Correlations among MALAT1, miR-145, and ADAMTS5 were analyzed by Spearman rank analysis. Chondrocytes viability and cartilage extracellular matrix (ECM) degradation were investigated with cell viability assay and Western blotting analyzing expression of ADAMTS5, collagen type 2 alpha 1 (COL2A1), aggrecan (ACAN), and cartilage oligomeric matrix protein (COMP).

Results: MALAT1 was upregulated, and miR-145 was downregulated in OA samples and IL-1 $\beta$-induced chondrocytes. Mechanically, miR-145 could directly bind to MALAT1 and ADAMTS5. Moreover, miR-145 expression was negatively correlated with MALAT1 and ADAMTS5 expression in OA patients, whereas MALAT1 and ADAMTS5 expression was positively correlated. Functionally, overexpression of MALAT1 inhibited chondrocyte viability and promoted cartilage ECM degradation in IL-1 $\beta$-induced chondrocytes. In support thereof, MALAT1 silencing and miR-145 upregulation exerted the opposite effect in IL-1 $\beta$-induced chondrocytes. Moreover, the effect of MALAT1 was counteracted by miR-145 upregulation, and ADAMTS5 restoration could abate miR-145 effects.

Conclusion: An MALAT1/miR-145 axis contributes to ECM degradation in IL- $1 \beta$-induced chondrocytes through targeting ADAMTS5, suggesting that MALAT1/miR-145/ADAMTS5 signaling may underlie human OA pathogenesis.

Key Words: MALAT1, miR-145, ADAMTS5, IL-1 $\beta$, osteoarthritis

\footnotetext{
Received: April 26, 2019 Revised: August 2, 2019 Accepted: August 14, 2019

Corresponding author: Yandong Wang, MD, Department of Orthopedics, the Forth Hospital of Yulin (Xingyuan Hospital), N0. 33 Renmin Road, West Yulin 71900, Shaanxi, China.

Tel: 86-0912-3264601, Fax: 86-0912-3264601, E-mail: rk6094246xiong@163.com

- The authors have no potential conflicts of interest to disclose.

(C) Copyright: Yonsei University College of Medicine 2019

This is an Open Access article distributed under the terms of the Creative Commons Attribution Non-Commercial License (https://creativecommons.org/licenses/by-nc/4.0) which permits unrestricted non-commercial use, distribution, and reproduction in any medium, provided the original work is properly cited.
} 


\section{INTRODUCTION}

Osteoarthritis (OA), also called chronic progressive joint disease ${ }^{1}$ or degenerative arthritis, ${ }^{2}$ is caused by various factors and may lead to chronic disability. OA is very common in older adults. The main characteristic of OA is the destruction of articular cartilage $^{3}$ due to the degeneration of cartilage extracellular matrix (ECM) and the loss of polyproteoglycans, such as a disintegrin and metalloproteinase with thrombospondin motifs (ADAMTS) 5, which belongs to the ADAMTS family. The family of aggrecanases comprises the main degradation enzymes of proteoglycans in cartilage matrix, ${ }^{5}$ of which ADAMTS5 is the major metalloproteinase $^{6}$ and positively correlated with articular cartilage degradation. ${ }^{7}$ Accordingly, gaining a deeper understanding of ${ }^{8}$ and searching for new molecular mechanisms and therapeutic strategies towards ADAMTS5 have become increasingly important.

MicroRNAs (miRNAs) are a series of small non-coding endogenous RNAs about 18-22 nucleotides in length ${ }^{9}$ that often have been found to negatively regulate protein-coding gene expression ${ }^{10}$ by commonly complementary binding to the 3 ' untranslated regions ( 3 ' UTR) of specific mRNA targets. ${ }^{9}$ Previous studies have indicated that miRNAs could be useful in searching diagnostic biomarkers, ${ }^{11}$ as well as providing novel therapeutic targets $^{12}$ for intervention in OA. For example, miRNA-145 (miR145) has been shown to regulate chondrocyte homeostasis. ${ }^{13}$ MiR-145 is implicated in cartilage dysfunction in OA. A previous study reported that miR-145 regulates MKK $4,{ }^{14} \mathrm{SMAD} 3,{ }^{13}$ Sox $9,{ }^{15}$ and TNFRSF $11 \mathrm{~B}^{16}$ expression by targeted binding in OA. However, whether miR-145 modulates ADAMTS5 expression by directly binding in OA tissues and cultured chondrocytes is unclear.

MiRNAs display functions in cellular events, which are ubiquitously mediated by long non-coding RNAs (lncRNAs) sponging. ${ }^{17}$ LncRNAs are a cluster of non-coding endogenous RNAs over 200 nucleotides in length ${ }^{18}$ and take part in regulating key cellular processes, ${ }^{11}$ such as proliferation, apoptosis, and differentiation. Recently, studies have been confirmed that lncRNA plays important roles in the development of inflammation-related diseases, ${ }^{19,20}$ such as rheumatoid arthritis, ${ }^{21}$ septicemia, ${ }^{22}$ and $\mathrm{OA} .{ }^{19}$ LncRNA metastasis-associated lung adenocarcinoma transcript 1 (MALAT1) has been implicated drug resistance ${ }^{23}$ and migration ${ }^{24}$ in cancer and has been found to have protective effects in cardiomyocytes ${ }^{25}$ and angiogenesis. ${ }^{26}$ Also, research has indicated that MALAT1/miRNA sponges exist extensively among cancers. ${ }^{27-31}$ However, the function of MALAT1 in OA has not been elucidated.

Cytokines are involved in the pathogenesis of $\mathrm{OA},{ }^{20}$ for example IL- $1 \beta$ and TNF- $\alpha$. High expression of IL- $1 \beta$ is usually involved in OA progression. ${ }^{32}$ Evidence further suggests that IL- $1 \beta$ regulates the expression of ADAMTS $4^{33}$ and ADAMTS $5^{34}$ in chondrocytes in OA. Compared with normal cartilage, ADAMTS5 is the most upregulated gene in human OA cartilage. ${ }^{35}$ Thus, there may a link between MALAT1, miR-145, and ADAMTS5 that has not yet been fully clarified.

In this study, we investigated the expression levels of MALAT1, miR-145, and ADAMTS5 in OA tissues and cells and correlations among them. MALAT1 upregulation attenuated cell viability and expression of cartilage ECM-related proteins collagen type 2 alpha 1 (COL2A1), aggrecan (ACAN) and cartilage oligomeric matrix protein (COMP) were accompanied by decreased miR145 expression. MiR-145 sequestered the effects of MALAT1 and ADAMTS5, likely by targeting binding. Our data suggest that a MALAT1/miR-145/ADAMTS5 axis could be an important pathway in OA progression, potentially holding novel clinical implications in clinical diagnosis of and treatment strategies for OA.

\section{MATERIALS AND METHODS}

\section{Patients and tissue samples}

This study was approved by the Research Ethics Committee of the Forth Hospital of Yulin (Xingyuan Hospital), and written informed consent was obtained from all patients. Cartilage tissues from 24 OA patients (age $65.8 \pm 8.5$ years) were obtained from the Forth Hospital of Yulin (Xingyuan Hospital). Normal cartilage tissues were collected from 11 patients (age $44.3 \pm 6.5$ years) who underwent amputation without a history of OA or rheumatoid arthritis. The diagnoses of these people were made according to the American College of Rheumatology criteria. Samples were immediately frozen and stored in liquid nitrogen.

\section{Cell line and culture}

Chondrocytes were isolated from OA cartilage tissues, as previously described. ${ }^{13}$ In brief, cartilage samples were cut into small chippings, followed by being subjected to digestion with $0.1 \%$ trypsin (Invitrogen, Carlsbad, CA, USA) for $30 \mathrm{~min}$ and then with $0.2 \%$ collagenase II (MilliporeSigma, Billerica, MA, USA) in highglucose Dulbecco's modified Eagle's medium (H-DMEM) (HyClone, Logan, UT, USA) for $10 \mathrm{~h}$ at $37^{\circ} \mathrm{C}$ with stirring every $1 \mathrm{~h}$. After centrifugation, chondrocytes were obtained and cultured in H-DMEM containing 10\% (v/v) fetal bovine serum (HyClone), and $1 \%$ antibiotics $(80 \mathrm{U} / \mathrm{mL}$ penicillin and $80 \mu \mathrm{g} / \mathrm{mL}$ streptomycin) (Invitrogen) at $37^{\circ} \mathrm{C}$ in an atmosphere of $5 \% \mathrm{CO}_{2}$. The primary culture was for 7 days, and secondary cultures were seeded onto 6-well plates (Corning, NY, USA) or 35-mm plates (Corning) for further study.

\section{Transient transfection}

For overexpression, MALAT1 and ADAMTS5 CDS were amplified and cloned into the multiple cloning site (MCS) of the pcDNA3.1 vector (Invitrogen). Small interfering RNA (siRNA) against MALAT1 (si-MALAT1), miR-145 mimics, and inhibitors were purchased from Ribobio Co. (Guangzhou, China). Sequences were as follows: si-MALAT1, 5'-GAGGUGUAAAGGGAU UUAUTT-3'; and si-MALAT1 \#2, 5'-AUAAAUCCCUUUACACC 


\section{UCTT-3'.}

Cell transfection with oligonucleotides or plasmids into chondrocytes was performed by Lipofectamine 2000 reagent (Invitrogen) according to the manufacturer's instructions. Cells were subsequently cultured about $48 \mathrm{~h}$ for further study.



Chondrocytes secondary cultured were exposed into medium containing recombinant human IL- $1 \beta$ (R\&D Systems, Minneapolis, MN, USA). Pretreatment with serum-free for $16 \mathrm{~h}$, chondrocytes were stimulated with $5,10 \mathrm{ng} / \mathrm{mL} \mathrm{IL}-1 \beta$ for $24 \mathrm{~h}$. The following experiments are based on the subline cells IL-1 $\beta$-stimulated chondrocytes, such as cell viability assay and Western blotting to detect functions of miR-145 and ADAMTS5.

\section{Dual-luciferase reporter assay}

Artificially synthesized MALAT1 wild-type and mutant-type (namely MALAT1-WT/MUT) and ADAMTS5 3' UTR wild-type and mutant-type (ADAMTS5-WT/MUT) constructs containing the putative binding sites of miR-145 were cloned into pGL3basic vectors (Promega, Madison, WI, USA) behind the luciferase gene, respectively. Following restriction endonuclease digestion, the target fragment was inserted into the pGL3 reporter plasmid. Chondrocytes were cultivated in 24-well plates (Corning) and co-transfected with either MALAT1-WT/MUT or ADAMTS5-WT/MUT and either miR-145/NC mimics or inhibitors for $48 \mathrm{~h}$. The plasmid phRL-tk (used as internal control) containing the Renilla luciferase gene was purchased from Promega and co-transfected with recombinant pGL3-basic vectors. Cells were collected and analyzed with a dual-luciferase reporter assay system (Promega), according to the manufacturer's instructions. The relative luciferase activity was the ratio of Firefly to Renilla luciferase activity.

\section{RNA isolation and quantitative real-time PCR}

Total RNA from OA tissues and cultured chondrocytes was extracted with TRIzol reagent (Thermo Fisher Scientific, Waltham, MA, USA) following the manufacturer's protocol. The concentration and purity of total RNA was examined using a NanoDrop 2000 spectrophotometer (Thermo Fisher Scientific).

Reverse transcription to cDNA depended on a reverse transcription kit (Abcam, Cambridge, UK). The amplification of cDNA was performed by SYBR Premix Ex Taq Master Mix $(2 \times)$ (Takara, Mountain View, CA, USA) supplemented with ROX reference Dye II on an Applied Biosystems 7500 Real-Time PCR System (Thermo Fisher Scientific). The reaction system $(20 \mu \mathrm{L})$ included 10.0 $\mu \mathrm{L}$ of SYBR Premix Ex Taq Master Mix $(2 \times), 0.8 \mu \mathrm{L}$ each of forward and reverse primers $(10 \mu \mathrm{M}), 2.0 \mu \mathrm{L}$ of cDNA ( $<500 \mathrm{ng}$ ), and $6.4 \mu \mathrm{L}$ of nuclease free- $\mathrm{H}_{2} \mathrm{O}$. The real-time PCR program was as follows: $95^{\circ} \mathrm{C}$ for $3 \mathrm{~min}$, followed by 40 cycles at $94^{\circ} \mathrm{C}$ for $15 \mathrm{~s}, 60^{\circ} \mathrm{C}$ for $30 \mathrm{~s}, 72^{\circ} \mathrm{C}$ for $20 \mathrm{~s}$, and then $4^{\circ} \mathrm{C}$ for $10 \mathrm{~min}$. The expression levels of MALAT1 and mature miR-145 were calculated by $2^{-\triangle \Delta C T}$ methods with normalization to glyceraldehyde- 3-phosphate dehydrogenase (GAPDH) and U6 small nuclear RNA (U6), respectively. Primers were as follows: MALAT $1^{36}$ : 5'-ATGCGAGTTGTTCTCCGTCT-3' (forward) and 5'-TATCTG CGGTTTCCTCAAGC-3' (Reserve); miR-145 $5^{37}$ : 5' -TCCAGTTT TCCCAGGAATCCCT-3' (forward) and 5'-CGCTTCACGAATT TGCGTGTCAT-3' (Reserve); GAPDH ${ }^{37}: 5^{\prime}$-GTCAACGGATTT GGTCTGTATT-3' (forward) and 5'-AGTCTTCTGGGTGGCAG TGAT-3' (Reserve); U6 ${ }^{37}$ : 5'-GCTTCGGCAGCACATATACTAA AAT-3' (forward) and 5'-CGCTTCACGAATTTGCGTGTCAT-3' (Reserve).

\section{Cell viability assay}

Cell viability was assayed using (4-5-dimethylthiazol-2-yl)-2, 5-diphenyl tetrazolium bromide (MTT) assay. ${ }^{38}$ Cultured chondrocytes with different treatments were incubated in 96-well plates (Corning) at a cell density of $1 \times 10^{5}$ cells per well in $150 \mu \mathrm{L}$ of medium for $24 \mathrm{~h}$ prior to insult with $5 \mathrm{mg} / \mathrm{mL}$ of MTT (Sigma-Aldrich, St. Louis, MO, USA) for another $4 \mathrm{~h}$, followed by the addition of $150 \mu \mathrm{L}$ of DMSO (Sigma-Aldrich) to dissolve the generated formazan crystals. Absorbance at a wavelength of 490 nm was detected using a microplate reader.

\section{Western blotting}

Total protein from cultured chondrocytes was extracted with RIPA Reagent (Beyotime Institute of Biotechnology, Jiangsu, China) and analyzed by Western blotting. The concentrations of total protein were measured with BCA reagent (Beyotime Institute of Biotechnology). $20 \mu$ g total protein was loaded on $8-10 \%$ sodium dodecyl sulfate polyacrylamide gel electrophoresis, and after $120 \mathrm{~min}$, proteins were transferred onto a polyvinylidene fluoride membrane (MilliporeSigma), which was blocked with $5 \%$ nonfat milk for another $2 \mathrm{~h}$ at room temperature or overnight at $4^{\circ} \mathrm{C}$. Then, membranes were incubated with primary antibody against ADAMTS5 (1:200 dilution; Sigma-Aldrich), COL2A1 (1:2500 dilution, Abcam, Cambridge, UK), ACAN (1:200 dilution, Abcam), COMP (1:200 dilution, Abcam), GAPDH (1:5000; Cell Signaling Technology, Danvers, MA, USA) overnight at $4^{\circ} \mathrm{C}$. GAPDH was used as an internal loading control. After being incubated with horseradish peroxidase-conjugated secondary antibody (Santa Cruz, Dallas, TX, USA) at room temperature for $1 \mathrm{~h}$, the membranes were visualized using enhanced chemiluminescence assay (MilliporeSigma) on a chemiluminescence instrument. The quantification analysis was conducted using Image J 1.8.0 (The National Institutes of Health, Bethesda, MD, USA).

\section{Statistical analysis}

Data are presented as means \pm SD. Statistical significance was determined by Mann-Whitney U test. The correlation between the expression of MALAT1, miR-145, and ADAMTS5 was examined by Spearman rank analysis using SPSS 16.0 software (SPSS Inc., Chicago, IL, USA). $p<0.05$ was considered significant. 


\section{RESULTS}

\section{MiR-145 expression is negatively correlated with MALAT1 in OA patients}

MiR-145 plays important roles in OA progression, ${ }^{13-15}$ and MALAT displays cellular functions by inhibiting miR-145 expression ${ }^{28-31}$ by target binding. To identify the potential role of MALAT1 and miR-145 in OA, we first checked their expression levels in samples derived from 11 normal tissues and $24 \mathrm{OA}$ cartilage tissues using quantitative real-time PCR (qRT-PCR). Compared with normal, MALAT1 expression was increased (Fig. 1A) in OA samples, while miR-145 was decreased (Fig. 1B). Also, the expression of MALAT1 was negatively correlated with miR-145 (Fig. 1C) as analyzed by Spearman rank analysis. These results suggest that MALAT1/miR-145 might be involved in OA.

\section{MALAT1 directly targets miR-145 in OA}

To further clarify the relationship between MALATl and miR-145, we used starBase (http://starbase.sysu.edu.cn/) to predict potential binding sites. As shown in Fig. 2A, the GGAAAACTGGAA region in MALAT1 was a candidate binding site of miR-145. To confirm this, the dual-luciferase reporter system was applied. As was shown in Fig. 2B, the relative luciferase activity decreased by about $60 \%$ in cells co-transfected with MALAT1-WT and miR-145 mimics, while there was no significant difference in MALAT1-MUT cells, compared to miR-NC mimics. Relative luciferase activity increased about $80 \%$ (Fig. 2C) in cells co-transfected with MALAT1-WT and miR-145 inhibitors, while there was no significant difference in MALAT1-MUT cells, compared to miR-NC inhibitors. Next, we checked whether MALAT1 could affect miR-145 expression. We used siRNA to knockdown MALAT1 in cultured chondrocytes (Fig. 2D) and found that miR-145 expression was upregulated over three-fold in cells treated with si-MALAT1 (Fig. 2E) using qRT-PCR. Instead, MALAT1 overexpression (Fig. 2F) could decrease miR-145 expression (Fig. 2G). These results demonstrated that MALAT1 inhibited miR-145 expressions through direct binding.
MALAT1 modulates cell viability and cartilage ECM degradation via miR-145 in IL-1 $\beta$-induced chondrocytes To explore the effect of MALAT1 in OA, we treated chondrocytes with $10 \mathrm{ng} / \mathrm{mL}$ of IL-1 $\beta$ to mimic human OA. ${ }^{7,13}$ First, MALAT1 and miR-145 expression induced by IL-1 $\beta$ was tested. As Fig. 3A and B showed, IL-1 $\beta$ stimulated MALAT1 upregulation and miR-145 downregulation simultaneously, and this affect was dose-dependent. Secondary, the effect of MALAT1 on cell viability was measured with MTT assay. MALAT1 impaired cell viability in IL-1 $\beta$-induced chondrocytes (Fig. 3C); conversely, siMALAT1 boosted chondrocytes viability. Also, the absorbance of si-MALAT1 chondrocytes and MALAT1 chondrocytes showed significant differences at $24 \mathrm{~h}, 48 \mathrm{~h}$, and $72 \mathrm{~h}$, compared with that in IL-1 $\beta$-induced chondrocytes (ctrl). Interestingly, upon co-transfection of miR-145 mimics, the cells exhibited higher cell viability (Fig. 3D) at $48 \mathrm{~h}$ and $72 \mathrm{~h}$ than MALAT1 cells. This suggested that miR-145 reversed the effect of MALAT1 on anti-proliferation in IL-1 $\beta$-induced chondrocytes. Considering that ADAMTS5, COL2A1, ACAN, and COMP are the biomarkers of cartilage ECM, we checked their expression by Western blotting. ADAMTS5 was upregulated in IL-1 $\beta$ treatment; while COL2A1, ACAN, and COMP were lower. MALAT1 induced ADAMTS5 expression, whereas si-MALAT reversed this greatly. Moreover, the effects of MALAT1 on COL2A1, ACAN, and COMP were in opposition to that on ADAMTS5 (Fig. 3E). The quantification and normalization of ADAMTS5, COL2A1, ACAN, and COMP expression levels were conducted on Image J software (Fig. 3F and G). Promisingly, miR-145 rescued ECM-related protein expressions induced by MALAT1 (Fig. 3H and I). ADAMTS5 expression was ameliorated with co-expression of miR-145 mimics. Similarly, COL2A1, ACAN, and COMP expression was restored by miR-145. These data indicated that overexpression of MALAT1 elicits the anti-proliferation and pro-degradation effects of ECM stimulated by IL- $1 \beta$, and the effects could be reversed by miR145. Further, we supposed that MALAT1 and IL-1 $\beta$ upregulate ADAMTS5 synergistically in OA.

\section{Expression correlation of ADAMTS5 with MALAT1} and miR-145 in OA samples

Expecting that ADAMTS5 would be promoted by MALAT1 and



A

Fig. 1. The roles of MALAT1 and miR-145 in osteoarthritis (OA) samples. (A and B) qRT-PCR showed increased MALAT1 and decreased miR-145 in OA samples, compared with that in normal cartilage tissues. (C) Spearman rank analysis revealed a negative correlation between MALAT1 and miR-145 expression. ${ }^{*} p<0.05$. 
overturned by miR-145, we wondered whether any correlation between ADAMTS5, MALAT1, and miR-145 would be discernible. Thus, expressions of MALAT1, miR-145, and ADAMTS5 were estimated in OA samples, and correlation analysis was performed. The results revealed a positive linear correlation
(Fig. 4B) between ADAMTS5 and MALAT1 and a negative linear correlation (Fig. 4A) between ADAMTS5 and miR-145. This indicated that MALAT1/ADAMTS5 plays a complicated and vital role in OA pathological mechanisms.

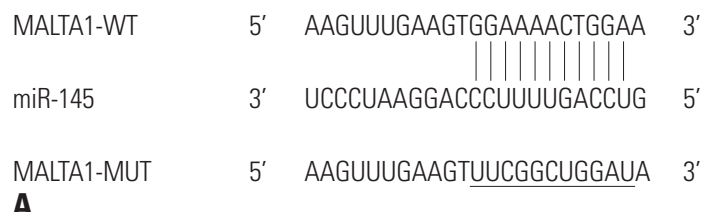

A
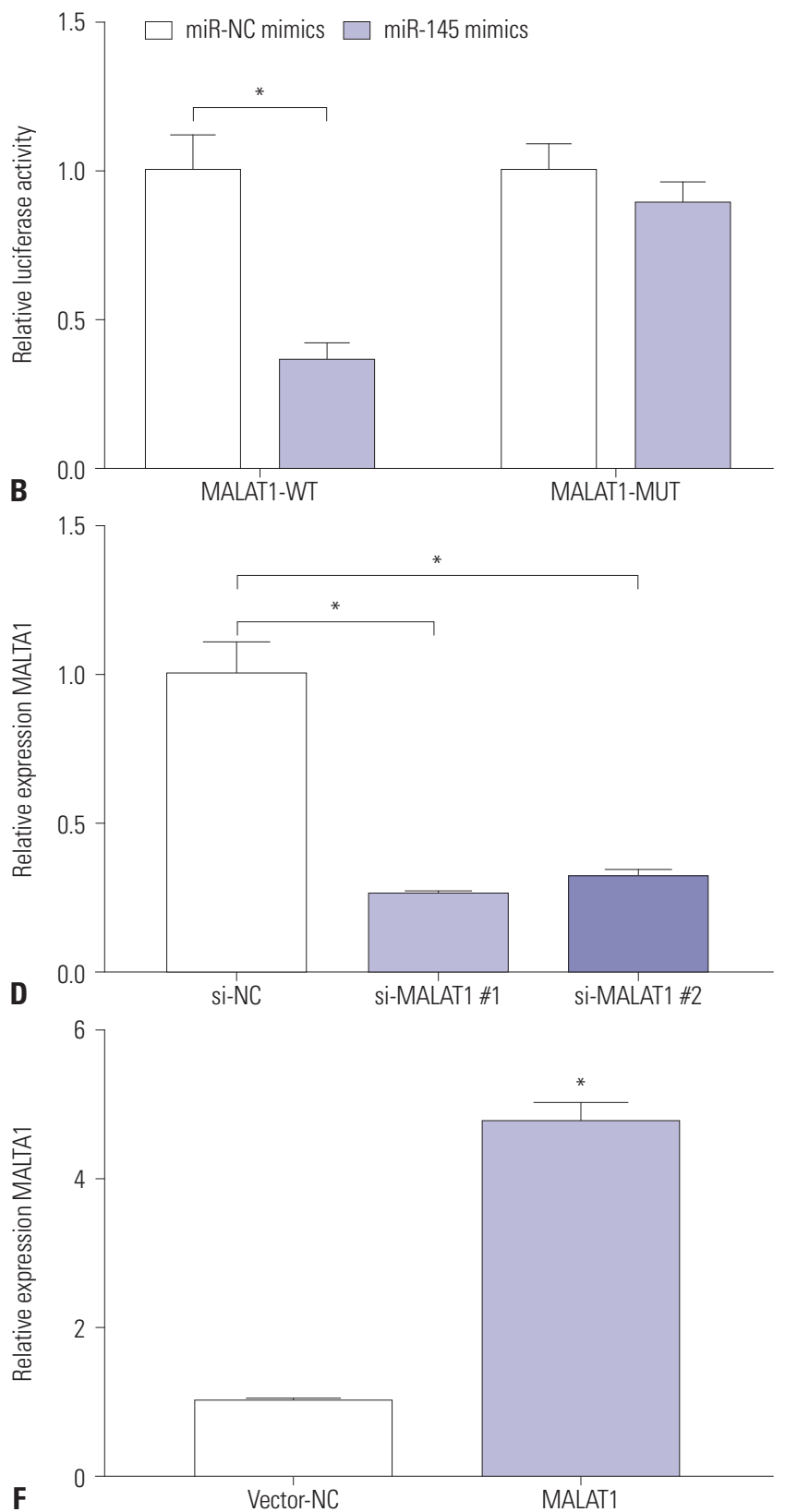


Fig. 2. MALAT1 attenuates miR-145 expression by target binding. (A) According to starBase, there was a conserved complementary binding site of miR145 in MALAT1. The mutant of MALAT1 wild-type (MALAT1-WT) is presented and named as MALAT1-MUT. (B and C) Transfection of miR-145 mimics/inhibitors distinctly decreased/facilitated the luciferase activity of MALAT1-WT chondrocytes. Expression level of MALAT1 and miR-145 in chondrocytes transfected with (D and E) siRNAs against MALAT1 (si-MALAT1 \#1 and 2) or (F and G) pcDNA3.1-MALAT1 (MALAT1) vector. Data represent means \pm SD. ${ }^{*} p<0.05$. 



Fig. 3. MALAT1 modulates IL-1 $\beta$-induced chondrocytes cell viability and cartilage ECM degradation via miR-145 in IL-1 $\beta$-induced chondrocytes. (A and B) Chondrocytes were treated with different concentrations of IL-1 $\beta$ for $24 \mathrm{~h}$, and expression of MALAT1 and miR-145 was detected using qRT-PCR. (C and D)


with miR-145 mimics. All data are presented as means \pm SD. * $p<0.05$. ECM, extracellular matrix.

\section{ADAMTS5 is a direct target of miR-145}

As we found MALAT1 suppresses miR-145 and promotes ADAMTS5 in OA tissues and/or IL-1 $\beta$-induced chondrocytes, we continued to research the interaction of miR-145 and ADAMTS5. First, as shown in Fig. 5A, ADAMTS5 and miR-145 owned two complementary combining regions in nature, and the GAUAACUGGA region and ACUGGA region in ADAMTS5 were candidate binding sites of miR-145. Accordingly, we mutated both regions in pGL3-basic. Subsequent dual-luciferase report assay validated that miR-145 targets and binds to ADAMTS5 (Fig. $5 \mathrm{~B}$ and $\mathrm{C}$ ). The interplay of each other was analyzed by Western blotting and qRT-PCR. ADAMTS5 protein (Fig. 5D and E) and mRNA levels (Fig. 5F and G) were reduced because of miR-145 mimics and elevated because of miR-145 knockdown. These results demonstrated that miR-145 sequesters ADAMTS5 through targeted binding.

\section{MALAT1/miR-145 modulates IL-1 $\beta$-induced chon- drocyte viability and cartilage ECM degradation via ADAMTS5}

MALAT1, miR-145, and ADAMTS5 worked with each other in a manner of linear dependence. More experiments were designed and conducted to identify the impact of MALAT1/miR-
145 on ADAMTS5 in IL-1 $\beta$-induced chondrocytes. Cell viability assay revealed that ADAMTS5 impairs miR-145 viability (Fig. 6A). Western blotting assay suggested that ADAMTS5 reduced COL2A1, ACAN, and COMP upregulation modulated by miR145 (Fig. 6B-D). Also, miR-145 downregulated ADAMTS5 and upregulated COL2A1, ACAN, and COMP in IL-1 $\beta$-induced chondrocytes (Fig. 6B-D). These tests demonstrated that ADAMTS5 affects MALAT1/miR-145 cellular functions, including cell viability and cartilage matrix homeostasis.

\section{DISCUSSION}

OA has always been one of the most leading non-fatal burden of life all over the world..$^{39}$ LncRNAs and miRNAs have garnered increasing attention in OA pathogenesis and progression recently. ${ }^{40,41}$ In this report, we identified MALAT1 expression as being higher and miR-145 expression as being lower in OA tissues and cultured cells. We also demonstrated that MALAT1 downregulates miR-145 likely via direct binding and is upregulated by IL-1 $\beta$ in chondrocytes. Meanwhile, miR-145 suppressed ADAMTS5 possibly by target binding and was inhibited by IL- $1 \beta$ in chondrocytes. Moreover, MALAT1 contributed to anti-proliferation 

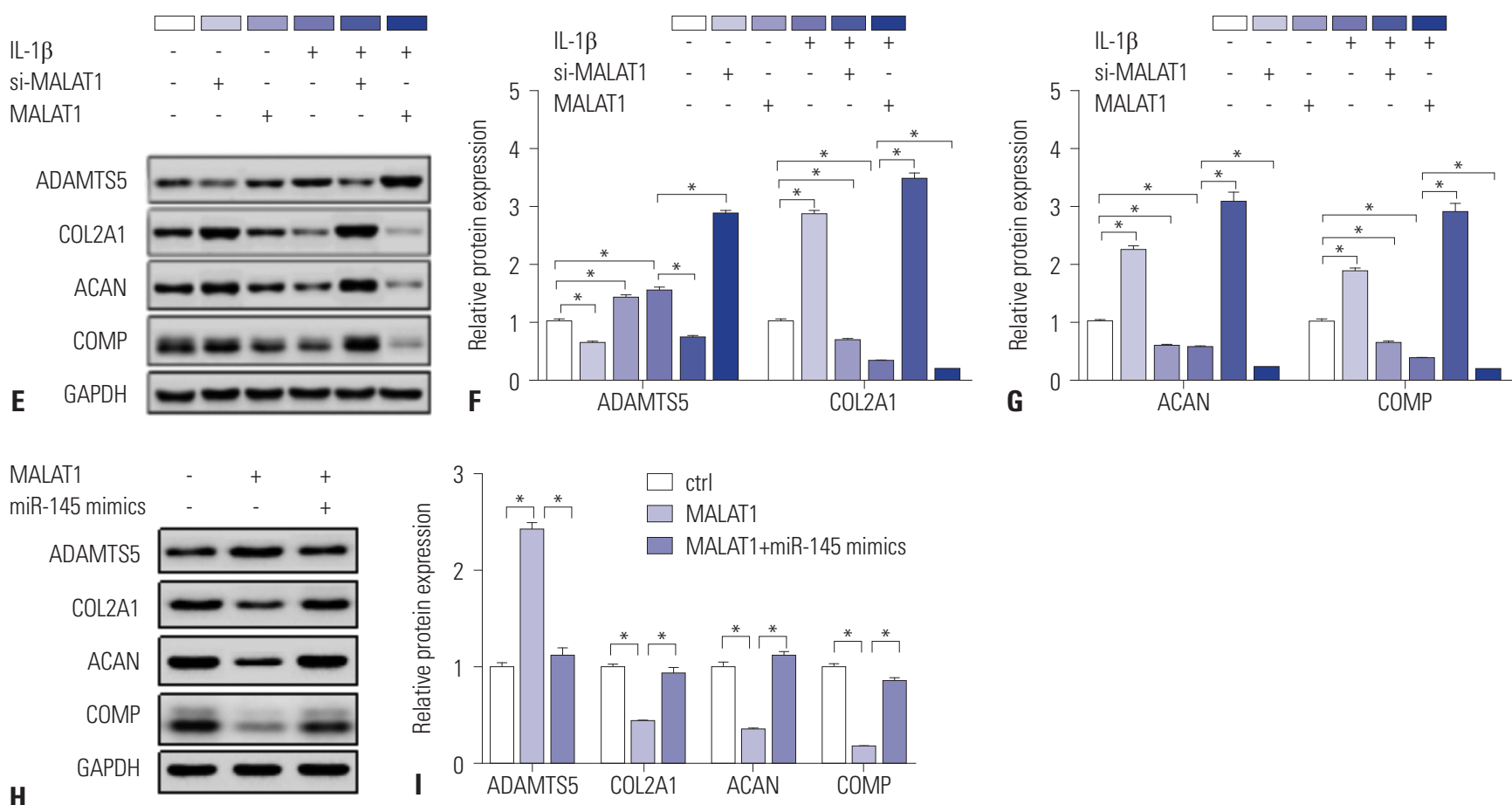


pression of ECM-related proteins was examined by Western blotting in chondrocytes transfected with si-MALAT1 \#1 and MALAT1 vector. (F and G) Quantitative analysis of ADAMTS5, COL2A1, ACAN, and COMP expressions using Image J. (H and I) Expression of ADAMTS5, COL2A1, ACAN, and COMP in IL-1 $\beta$-induced chondrocytes transfected with MALAT1 vector only or combined with miR-145 mimics. All data are presented as means \pm SD. ${ }^{*} p<0.05$. ECM, extracellular matrix.
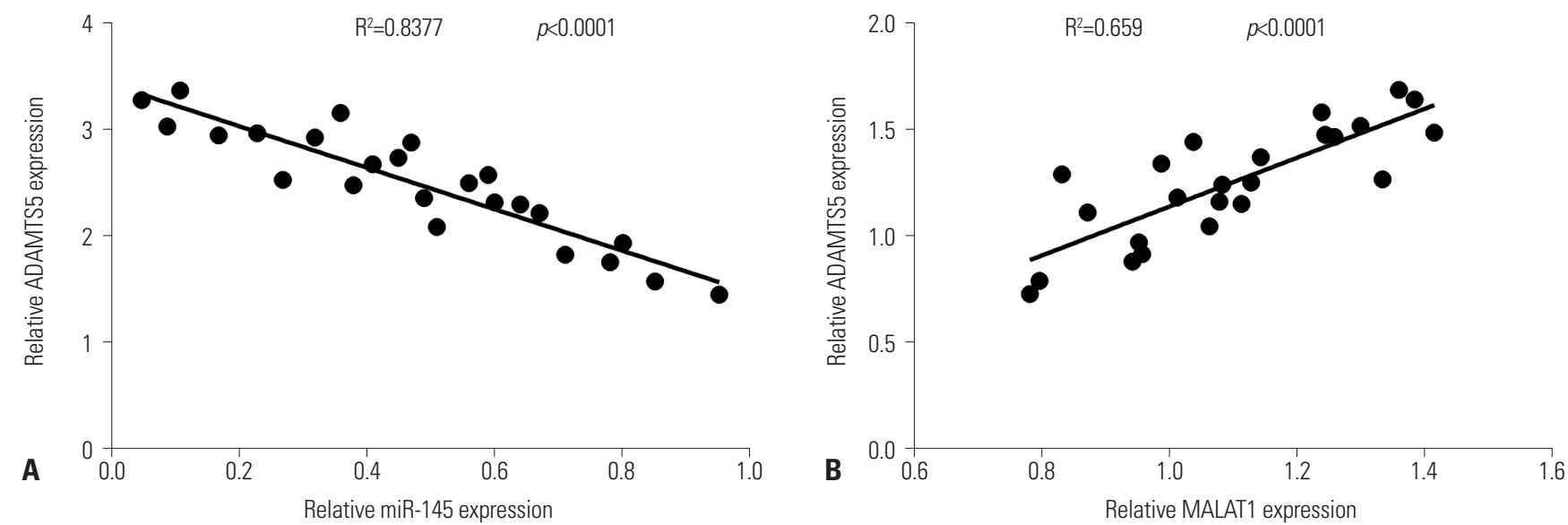

Fig. 4. Correlation analysis between expression levels of ADAMTS5, MALAT1, and miR-145. Spearman rank analysis revealed (A) a negative correlation between ADAMTS5 and miR-145 expression and (B) a positive correlation between ADAMTS5 and MALAT1 expression.

and cartilage ECM degradation, which was reversed by miR-145: miR-145 protected cell viability and cartilage matrix stability, which was canceled by ADAMTS5. In conclusion, MALAT1 modulated IL-1 $\beta$-induced chondrocyte viability and cartilage ECM degradation via miR-145/ADAMTS5 axis.

Accumulating evidence suggests that multiple lncRNAs are involved in the pathogenesis of OA. For example, synovial fluid-derived exosomal IncRNA PCGEM1 ${ }^{42}$ was shown to be a biomarker for analyzing the different stages of OA. MALAT1 has been shown to affect drug resistance and migration in cancer ${ }^{23,43}$ and to exert protective effects in cardiomyocyte ${ }^{25}$ and angio- genesis. ${ }^{26,44}$ However, there have been no research investigating the role of MALAT1 in OA. In our study, we found that MALAT1 was detrimental to cell viability and aggravated cartilage matrix degradation in OA. Overexpression of MALATl attenuated the cell viability, and knockdown of MALAT1 greatly improved cell viability in cultured chondrocytes. Meanwhile, overexpression of MALAT1 induced greater levels of ADAMTS5, COL2A1, ACAN, and COMP, compared with those stimulated by IL-1 $\beta$. Knockdown of MALAT1 negatively regulated both $\mathrm{IL}$-1 $\beta$-mediated ADAMTS5 and non-IL-1 $\beta$-mediated ADAMTS5 in chondrocytes. In addition, knockdown of MALAT1 positively regulated both 


$\begin{array}{lcclll}\text { ADAMTS5-WT } & 5^{\prime} & \text {...CUUAUGCACAAAGAUAACUGGAG... } & \text { ADAMTS5-WT } & 5^{\prime} & \text {...AGCUGUGGAUAUUUUACUGGAAA... } \\ \text { miR-145 } & 3^{\prime} & \text { UCCCUAAGGACCCUUUUGACCUG } & \text { miR-145 } & 3^{\prime} & \text { UCCCUAAGGACCCUUUUGACCUG } \\ \text { ADAMTS5-MUT } & 5^{\prime} & \text {...CUUAUGCACAAAACUUCAAUCCG.... } & \text { ADAMTS5-MUT } & 5^{\prime} & \text {...AGCUGUGGAUAUUUCGAAUCAA.... }\end{array}$

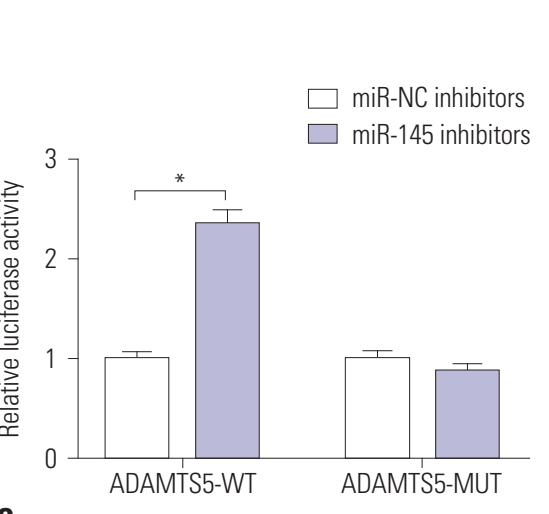

B
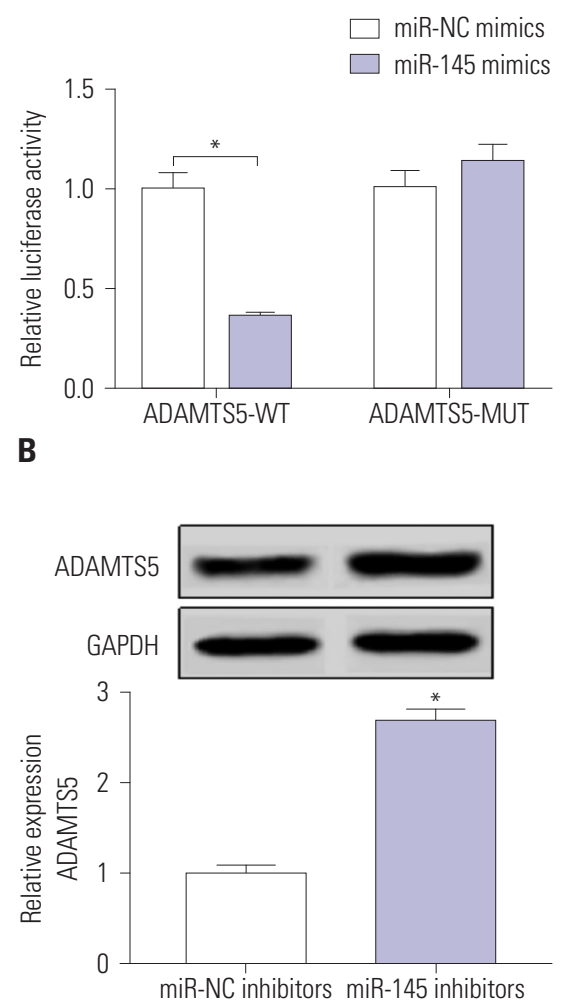

C

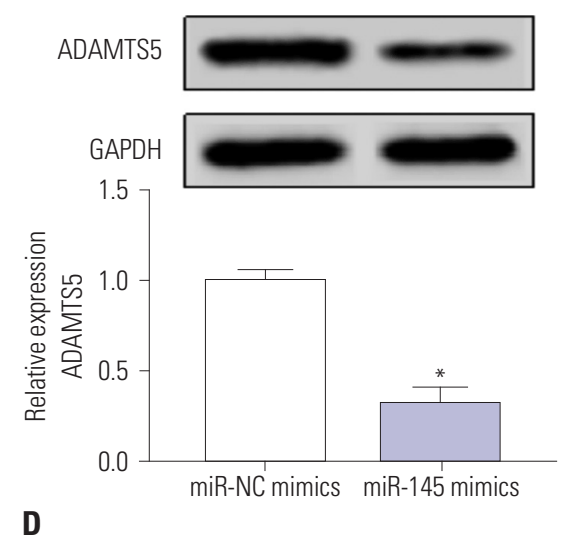

\section{E}

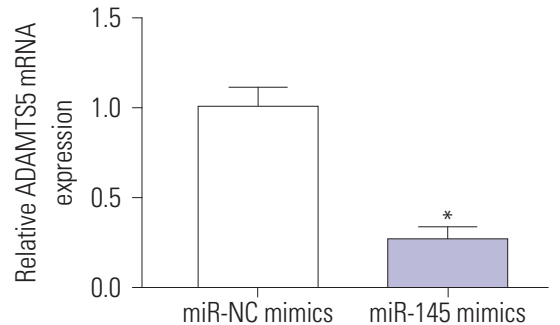

$\mathbf{F}$

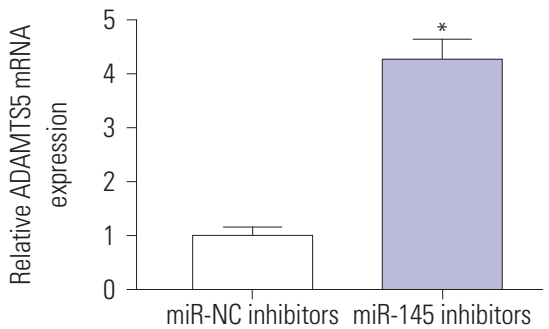

G

Fig. 5. miR-145 might sequester ADAMTS5 by target binding. (A) The databases TargetScan (http://www.targetscan.org/) and miRcode (http://www.mircode.org/) showed two conserved binding sites of miR-145 in the 3' UTR of ADAMTS5. The wild-type of ADAMTS5 (ADAMTS5-WT) was mutated as MALAT1-MUT. (B, C) Relative luciferase activity of ADAMTS5-WT/MUT was determined by luciferase reporter assay in chondrocytes when co-transfected with miR-145 mimics or inhibitors. (D, E) Western blotting detected ADAMTS5 protein expression level after chondrocytes transfected with miR145 mimics or inhibitors. $(F, G)$ qRT-PCR detected ADAMTS5 mRNA expression level after chondrocytes transfected with miR-145 mimics or inhibitors. Data are presented as means \pm SD. ${ }^{*} p<0.05$.

IL-1 $\beta$-mediated and non-IL-1 $\beta$-mediated COL2Al, ACAN, and COMP in chondrocytes.

MiR-145 plays a vital role in arthritic chondrocytes. In previous studies, miR-145 was found to act as a TNF- $\alpha$-responsive miRNA in chondrocytes, ${ }^{14}$ to regulate radiosensitivity of HR-HPV+ cervical cancer, ${ }^{28}$ to enhance docetaxel-resistance of prostate cancer, ${ }^{29}$ to promote vascular endothelial cell angiogenesis, ${ }^{44}$ and to protect against oxygen-glucose deprivation-induced apoptosis. According to this study, miR-145 upregulation rescued and canceled the anti-proliferation effect of MALAT1 in chondrocytes. We observed that cell viability was dramatically decreased when chondrocytes overexpressed MALATl, which was significantly elevated when chondrocytes co-overexpressed of MALAT1 and miR-145. Additionally, there was no difference be- tween chondrocytes co-overexpressing MALAT1 and miR-145 and normal chondrocytes. We proposed that miR-145 restrained cartilage matrix degradation by turning down ADAMTS5 and turning up COL2A1, ACAN, and COMP. Recently, a few miRNAs, ${ }^{33,45,46}$ including miR-146a, miR-148a, and miR-140, have been shown to be involved in the regulation of ADAMTS5 expression. Some evidence has suggested that miR- $145^{14}$ attenuates TNF- $\alpha$ induced cartilage matrix degradation by downregulating $\mathrm{AD}$ AMTS5 and MMP3/13, showing no significant difference on COL2A1, SOX9, and ACAN. Another study ${ }^{15}$ showed that miR-145 reduced COL2A1 and ACAN and increased RUNX2 and MMP13 to regulate chondrocyte functions. Meanwhile, the expression levels of miR-145 in OA tissues has remained controversial, ${ }^{13,14,16,31}$ compared with normal articular cartilage tissues. Our data sup- 

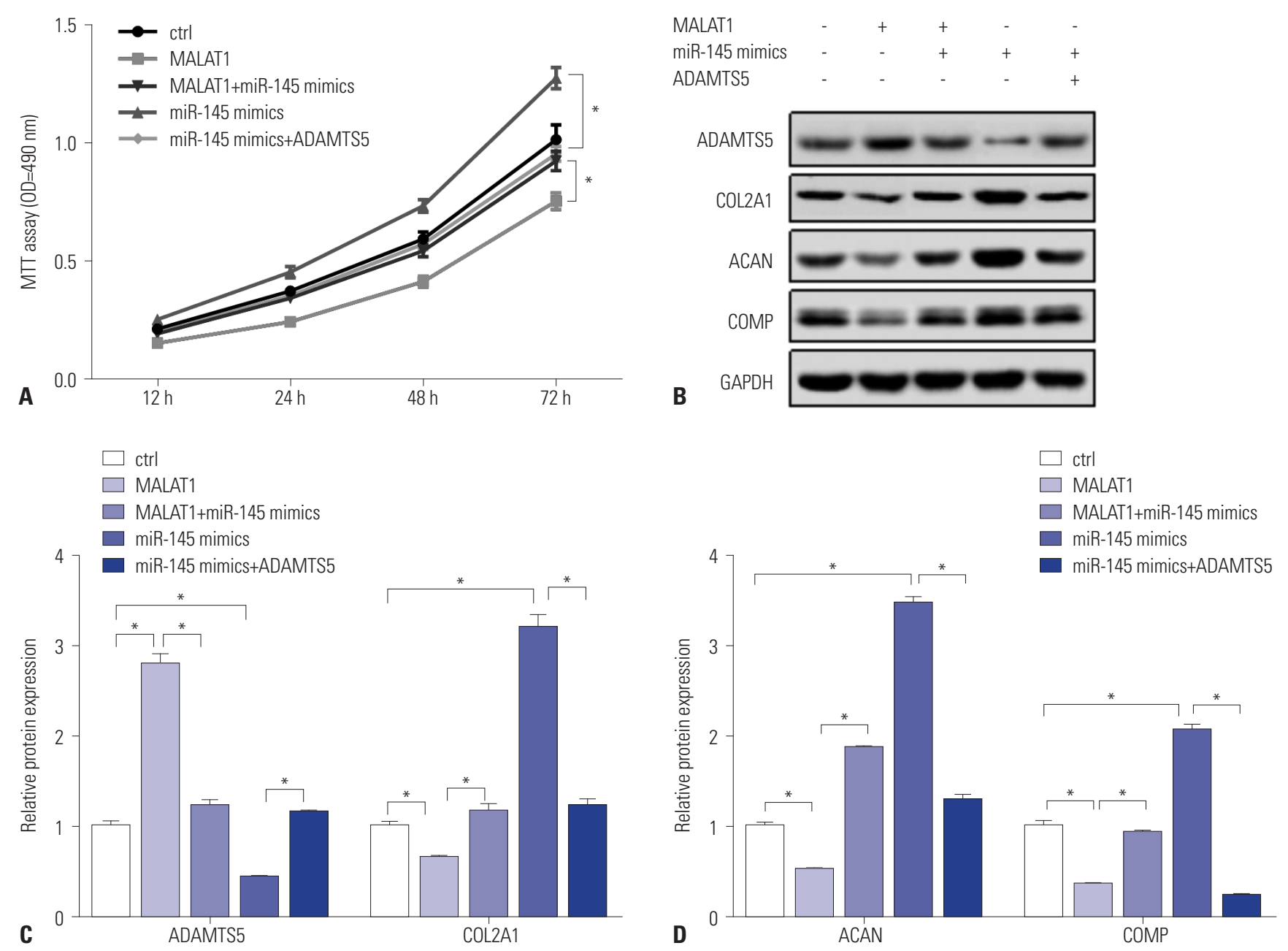

Fig. 6. ADAMTS5 modulates IL-1ß-induced chondrocyte viability and cartilage degradation mediated by MALAT1/miR-145. Chondrocytes were transfected with MALAT1 only or together with miR-145 mimics and miR-145 mimics only or combined with ADAMTS5 vector. After $10 \mathrm{ng} / \mathrm{mL}$ of IL-1 13 treatment, (A) cell viability was detected by MTT assay at $12 \mathrm{~h}, 24 \mathrm{~h}, 48 \mathrm{~h}$, and $72 \mathrm{~h}$, and (B) ECM-related protein expression was measured by Western blotting at $24 \mathrm{~h}$. (C and D) Quantitative analysis of ADAMTS5, COL2A1, ACAN, and COMP expression was conducted by Image J. All data are presented as means \pm SD. ${ }^{*} p<0.05$.

port the idea of downregulation of miR-145, which is consistent with studies by $\mathrm{Hu}$, et al. ${ }^{14}$ and Wang, et al. ${ }^{16}$ and in opposition to that by Yang, et al. ${ }^{13}$

MALAT1/miRNA sponges have been reported in cancers: for instance, MALAT1 $/ \mathrm{miR}-663^{47}$ in colon cancer, MALAT1/miR$211^{48}$ in ovarian carcinoma, and MALAT1/miR- $142-3 \mathrm{p}^{43}$ in cervical cancer. MALAT1 targeting miR-145 has been well-discussed in different diseases, including cancer and fibrosis. With bioinformatics tools, potential targets of a MALAT1/miR-145 axis have been described and further confirmed using luciferase reporter assay, RNA immunoprecipitation, and RNA pull-down assay. For example, downregulating the MALAT1/miR-145 axis targeting ZEB2 inhibited expression of $\alpha$-SMA, fibronectin, and collagen I in human kidney-2 (HK2) cells under high glucose treatment, which eventually resulted in tubulointerstitial fibrosis and glomerulosclerosis. ${ }^{49}$ MALAT1 knockdown attenuated angiotensin II-mediated cardiac fibrosis of infarcted hearts, as evidenced by decreases in $\alpha$-SMA and collagen I and III, by directly binding to miR- $145 .{ }^{50}$ However, there is no information about MALAT1/miR-145 axis in OA. This was why we planned to explore the action of MALAT1/miR-145 sponge in OA chondrocytes. In this study, we searched the putative binding site between MALAT1 and miR-145 on starBase software and further identified this target relationship utilizing luciferase reporter assay. Promisingly, we verified our hypothesis that miR-145 played important roles in chondrocyte viability and matrix homeostasis by MALAT1 sponging.

MiR-145 directly binds to ADAMTS5 to regulate cartilage matrix. Recently, a few miRNAs, ${ }^{33,45,46}$ including miR-146a, miR-148a and miR-140, have been shown to be involved in the regulation of ADAMTS5 expression. Previous studies have reported miR-145 targets at MKK4,${ }^{14}$ SMAD $3,{ }^{31}$ SEMA3A, ${ }^{51}$ and TNFRSF $11 B^{16}$ in arthritis, including $\mathrm{OA}$ and rheumatoid arthritis. Thus, we assumed that miR-145 directly acts on ADAMTS5 in OA, rather than via SOX9. ${ }^{15}$ Consistent with this expectation, the luciferase activity was decreased in chondrocytes co-expressing miR-145 
and wild-type ADAMTS5 and increased dramatically in chondrocytes co-expressing miR-145 and mutated ADAMTS5. The outcomes revealed that miR-145 likely targets ADAMTS5 to regulate IL-1 $\beta$-stimulated chondrocytes and functions with $\mathrm{AD}$ AMTS5 in a manner of negative linear dependence.

IL-1 $\beta$, in turn, modulated MALAT1 and miR-145 expression in chondrocytes. Studies have shown that cytokines are involved in the pathogenesis of $\mathrm{OA},{ }^{20}$ including IL- $1 \beta$ and TNF- $\alpha$. Evidence suggests that IL- $1 \beta$ regulate the expressions of ADAMTS $4^{33}$ and ADAMTS $5^{34}$ in chondrocytes in OA. In one study, miR-145 acted as a TNF- $\alpha$-responsive miRNA in chondrocytes. ${ }^{14}$ We noticed that MALAT1 was upregulated and that miR-145 was down-regulated in the presence of IL-1 $\beta$, to a certain extent in a concentration-dependent manner. Hong, et al. ${ }^{51}$ reported that miR-145 rendered sensitivity of synoviocytes to stimuli of TNF- $\alpha$ and VEGF. Li, et al. ${ }^{52}$ confirmed that miR-145 promoted the expression of IL- $1 \beta$ and TNF- $\alpha$ in atherosclerosis. Overall, MALAT1/ miR-145/ADAMTS5 appear to comprise a complicated and intertwining network in IL-1 $\beta$-stimulated OA.

Regrettably, these data lack the examination of ADAMTS5 expression in OA samples derived from patients. Still, the protein expression levels of ADAMTS5 were detected in cultured OA chondrocytes and were higher, which is identical with that in previous studies. Also, it is well known that ADAMTS5 expression is high in OA tissues and cells. Further investigations are required to validate the direct binding of miR-145 with MALAT1 and ADAMTS5. Nevertheless, our data were in agreement with previous descriptions of the interplay of MALAT1 and miR-145, which is target-binding to each other. Notably, we observed luciferase mRNA expression changes were similar to that of protein levels (data not shown), suggesting that miR-145 might begin to inhibit MALAT1 and ADAMTS5 at the transcription level. This hypothesis remains to be further investigated and confirmed, as well as the regulatory mechanism underlying miR145 in modulating MALAT1 and ADAMTS5.

Because the self-renewal of articular cartilage is extremely limited, ${ }^{53}$ most clinical and research efforts have focused on the restoration of cartilage damage in connection with OA or trauma. First and foremost, we pointed out the effect of MALAT1 in OA pathogenesis and the molecular mechanism of upregulating miR-145 targeting ADAMTS5. This research suggested that MALAT1/miR-145/ADAMTS5 could be a novel pathway of OA pathogenesis and a potential therapeutic strategy in clinical diagnosis and treatment of human OA by MALAT1 knockdown and/or miR-145 overexpression. Based on previous evidence and our present experiments, we speculate that MALAT1 and miR-145 could be promising therapeutic biomarkers to impede, stop, and even reverse OA progression by MALAT1 knockdown and/or miR-145 overexpression. Hence, it seems to be essential and indispensable to transfect lv-sh-MALAT1 into mouse cartilage chondrocytes. ${ }^{54}$ Experiments should be conducted to trace and monitor OA symptoms and progression.

In summary, MALAT1 was upregulated in OA tissues and cul- tured cells, and MALAT1 knockdown contributed to cell viability and matrix stability in IL-1 $\beta$-stimulated chondrocytes. ADAMTS5, COL2A1, ACAN, and COMP together resulted in cartilage matrix degradation and the loss of chondrocyte activity. MALAT1 could bind to miR-145 to regulate ADAMTS5 expression. Overexpression of MALAT1 repressed cell viability and improved ADAMTS5, which either MALAT1 knockdown or miR-145 overexpression could reverse. Upregulation of miR-145 advanced cell viability and reduced ADAMTS5 expression, which overexpression of ADAMTS5 could overturn. Collectively, this study demonstrated that miR-145 reverses MALAT1-mediated ECM degradation and anti-proliferation by inhibiting ADAMTS5 in IL-1 $\beta$-induced chondrocytes. This MALAT1/miR-145/ADAMTS5 regulatory axis provides a new theoretical basis for research on lncRNA-directed therapeutics in OA.

\section{ACKNOWLEDGEMENTS}

This study was supported by Teacher research fund support project of Jining Medical University (JYFC2018FKJ146).

\section{AUTHOR CONTRIBUTIONS}

Conceptualization: Chengyao Liu, Shan Ren. Data curation: Shifeng Zhao, Yandong Wang. Formal analysis: Yandong Wang. Funding acquisition: Shifeng Zhao. Investigation: Chengyao Liu. Methodology: Shan Ren. Project administration: Shifeng Zhao. Resources: Yandong Wang. Software: Yandong Wang, Chengyao Liu. Supervision: Chengyao Liu. Validation: Yandong Wang. Visualization: Shifeng Zhao. Writing_original draft: Yandong Wang. Writing_review \& editing: Chengyao Liu.

\section{ORCID iDs}

Chengyao Liu

Shan Ren

Shifeng Zhao

Yandong Wang

https://orcid.org/0000-0001-9127-1778 https://orcid.org/0000-0001-9668-8249 https://orcid.org/0000-0002-9947-7727 https://orcid.org/0000-0002-5274-0022

\section{REFERENCES}

1. Peters AE, Akhtar R, Comerford EJ, Bates KT. The effect of ageing and osteoarthritis on the mechanical properties of cartilage and bone in the human knee joint. Sci Rep 2018;8:5931.

2. Zhao Y, Zhao J, Guo X, She J, Liu Y. Long non-coding RNA PVT1, a molecular sponge for miR-149, contributes aberrant metabolic dysfunction and inflammation in IL-1 $\beta$-simulated osteoarthritic chondrocytes. Biosci Rep 2018;38:BSR20180576.

3. Akkiraju $\mathrm{H}$, Nohe A. Role of chondrocytes in cartilage formation, progression of osteoarthritis and cartilage regeneration. J Dev Biol 2015;3:177-92.

4. Mead TJ, Apte SS. ADAMTS proteins in human disorders. Matrix Biol 2018;71-72:225-39.

5. Tío L, Martel-Pelletier J, Pelletier JP, Bishop PN, Roughley P, Farran A, et al. Characterization of opticin digestion by proteases involved in osteoarthritis development. Joint Bone Spine 2014;81: 137-41. 
6. Verma P, Dalal K. ADAMTS-4 and ADAMTS-5: key enzymes in osteoarthritis. J Cell Biochem 2011;112:3507-14.

7. Ji Q, Xu X, Zhang Q, Kang L, Xu Y, Zhang K, et al. The IL-1ß/AP-1/ miR-30a/ADAMTS-5 axis regulates cartilage matrix degradation in human osteoarthritis. J Mol Med (Berl) 2016;94:771-85.

8. Ji Q, Xu X, Xu Y, Fan Z, Kang L, Li L, et al. miR-105/Runx2 axis mediates FGF2-induced ADAMTS expression in osteoarthritis cartilage. J Mol Med (Berl) 2016;94:681-94.

9. Fiorucci G, Chiantore MV, Mangino G, Percario ZA, Affabris E, Romeo G. Cancer regulator microRNA: potential relevance in diagnosis, prognosis and treatment of cancer. Curr Med Chem 2012;19: 461-74.

10. Kuninty PR, Schnittert J, Storm G, Prakash J. MicroRNA targeting to modulate tumor microenvironment. Front Oncol 2016;6:3.

11. Nugent M. MicroRNAs: exploring new horizons in osteoarthritis. Osteoarthritis Cartilage 2016;24:573-80.

12. Cong L, Zhu Y, Tu G. A bioinformatic analysis of microRNAs role in osteoarthritis. Osteoarthritis Cartilage 2017;25:1362-71.

13. Yang B, Kang X, Xing Y, Dou C, Kang F, Li J, et al. Effect of microRNA-145 on IL-1 $\beta$-induced cartilage degradation in human chondrocytes. FEBS Lett 2014;588:2344-52.

14. Hu G, Zhao X, Wang C, Geng Y, Zhao J, Xu J, et al. MicroRNA-145 attenuates TNF- $\alpha$-driven cartilage matrix degradation in osteoarthritis via direct suppression of MKK4. Cell Death Dis 2017;8:e3140.

15. Martinez-Sanchez A, Dudek KA, Murphy CL. Regulation of human chondrocyte function through direct inhibition of cartilage master regulator SOX9 by microRNA-145 (miRNA-145). J Biol Chem 2012;287:916-24.

16. Wang GD, Zhao XW, Zhang YG, Kong Y, Niu SS, Ma LF, et al. Effects of miR-145 on the inhibition of chondrocyte proliferation and fibrosis by targeting TNFRSF11B in human osteoarthritis. Mol Med Rep 2017;15:75-80.

17. Song X, Cao G, Jing L, Lin S, Wang X, Zhang J, et al. Analysing the relationship between lncRNA and protein-coding gene and the role of lncRNA as ceRNA in pulmonary fibrosis. J Cell Mol Med 2014;18:991-1003.

18. Veneziano D, Di Bella S, Nigita G, Laganà $A$, Ferro A, Croce CM. Noncoding RNA: current deep sequencing data analysis approaches and challenges. Hum Mutat 2016;37:1283-98.

19. Jiang SD, Lu J, Deng ZH, Li YS, Lei GH. Long noncoding RNAs in osteoarthritis. Joint Bone Spine 2017;84:553-6.

20. Marques-Rocha JL, Samblas M, Milagro FI, Bressan J, Martínez JA, Marti A. Noncoding RNAs, cytokines, and inflammation-related diseases. FASEB J 2015;29:3595-611.

21. Messemaker TC, Frank-Bertoncelj M, Marques RB, Adriaans A, Bakker AM, Daha N, et al. A novel long non-coding RNA in the rheumatoid arthritis risk locus TRAF1-C5 influences C5 mRNA levels. Genes Immun 2016;17:85-92.

22. Ho J, Chan H, Wong SH, Wang MH, Yu J, Xiao Z, et al. The involvement of regulatory non-coding RNAs in sepsis: a systematic review. Crit Care 2016;20:383.

23. Yuan P, Cao W, Zang Q, Li G, Guo X, Fan J. The HIF-2 $\alpha$-MALAT1miR-216b axis regulates multi-drug resistance of hepatocellular carcinoma cells via modulating autophagy. Biochem Biophys Res Commun 2016;478:1067-73.

24. Lee NK, Lee JH, Ivan C, Ling H, Zhang X, Park CH, et al. MALAT1 promoted invasiveness of gastric adenocarcinoma. BMC Cancer 2017;17:46.

25. Uchida S, Dimmeler S. Long noncoding RNAs in cardiovascular diseases. Circ Res 2015;116:737-50.

26. Thum T, Fiedler J. LINCing MALAT1 and angiogenesis. Circ Res 2014;114:1366-8.

27. Wang SH, Zhang WJ, Wu XC, Zhang MD, Weng MZ, Zhou D, et al.
Long non-coding RNA Malat1 promotes gallbladder cancer development by acting as a molecular sponge to regulate miR-206. Oncotarget 2016;7:37857-67.

28. Lu H, He Y, Lin L, Qi Z, Ma L, Li L, et al. Long non-coding RNA MALAT1 modulates radiosensitivity of HR-HPV+ cervical cancer via sponging miR-145. Tumour Biol 2016;37:1683-91.

29. Xue D, Lu H, Xu HY, Zhou CX, He XZ. Long noncoding RNA MALAT1 enhances the docetaxel resistance of prostate cancer cells via miR-145-5p-mediated regulation of AKAP12. J Cell Mol Med 2018;22:3223-37.

30. Zhao ZH, Hao W, Meng QT, Du XB, Lei SQ, Xia ZY. Long noncoding RNA MALAT1 functions as a mediator in cardioprotective effects of fentanyl in myocardial ischemia-reperfusion injury. Cell Biol Int 2017;41:62-70.

31. Xiang Y, Zhang Y, Tang Y, Li Q. MALAT1 modulates TGF- $\beta 1$ induced endothelial-to-mesenchymal transition through downregulation of miR-145. Cell Physiol Biochem 2017;42:357-72.

32. Starodubtseva IA, Vasilieva LV, Nikitin AV. [Correction of an inflammatory process with an interleukin-1 inhibitor in the combination treatment of secondary osteoarthritis in the presence of comorbid condition]. Ter Arkh 2015;87:41-8.

33. Li X, Zhen Z, Tang G, Zheng C, Yang G. MiR-29a and MiR-140 protect chondrocytes against the anti-proliferation and cell matrix signaling changes by IL-1 $\beta$. Mol Cells 2016;39:103-10.

34. Liu Q, Zhang X, Dai L, Hu X, Zhu J, Li L, et al. Long noncoding RNA related to cartilage injury promotes chondrocyte extracellular matrix degradation in osteoarthritis. Arthritis Rheumatol 2014; 66:969-78.

35. Dou P, Hu R, Zhu W, Tang Q, Li D, Li H, et al. Long non-coding RNA HOTAIR promotes expression of ADAMTS- 5 in human osteoarthritic articular chondrocytes. Pharmazie 2017;72:113-7.

36. Wang SH, Zhang WJ, Wu XC, Weng MZ, Zhang MD, Cai Q, et al. The lncRNA MALAT1 functions as a competing endogenous RNA to regulate MCL-1 expression by sponging miR-363-3p in gallbladder cancer. J Cell Mol Med 2016;20:2299-308.

37. Gao M, Miao L, Liu M, Li C, Yu C, Yan H, et al. miR-145 sensitizes breast cancer to doxorubicin by targeting multidrug resistanceassociated protein-1. Oncotarget 2016;7:59714-26.

38. Lu S, Xiao X, Cheng M. Matrine inhibits IL-1 $\beta$-induced expression of matrix metalloproteinases by suppressing the activation of MAPK and NF- $\kappa B$ in human chondrocytes in vitro. Int J Clin Exp Pathol 2015;8:4764-72.

39. Brody JA, Grant MD. Age-associated diseases and conditions: implications for decreasing late life morbidity. Aging (Milano) 2001; 13:64-7.

40. Sun T, Yu J, Han L, Tian S, Xu B, Gong X, et al. Knockdown of long non-coding RNA RP11-445H22.4 alleviates LPS-induced injuries by regulation of MiR-301a in osteoarthritis. Cell Physiol Biochem 2018;45:832-43.

41. Shen H, Wang Y, Shi W, Sun G, Hong L, Zhang Y. LncRNA SNHG5/ miR-26a/SOX2 signal axis enhances proliferation of chondrocyte in osteoarthritis. Acta Biochim Biophys Sin (Shanghai) 2018;50: 191-8.

42. Zhao Y, Xu J. Synovial fluid-derived exosomal lncRNA PCGEM1 as biomarker for the different stages of osteoarthritis. Int Orthop 2018;42:2865-72.

43. Xia C, Liang S, He Z, Zhu X, Chen R, Chen J. Metformin, a firstline drug for type 2 diabetes mellitus, disrupts the MALAT1/miR$142-3 p$ sponge to decrease invasion and migration in cervical cancer cells. Eur J Pharmacol 2018;830:59-67.

44. Ren L, Wei C, Li K, Lu Z. LncRNA MALAT1 up-regulates VEGF-A and ANGPT2 to promote angiogenesis in brain microvascular endothelial cells against oxygen-glucose deprivation via target- 
ting miR-145. Biosci Rep 2019;39:BSR20180226.

45. Gu SX, Li X, Hamilton JL, Chee A, Kc R, Chen D, et al. MicroRNA146a reduces IL-1 dependent inflammatory responses in the intervertebral disc. Gene 2015;555:80-7.

46. Vonk LA, Kragten AH, Dhert WJ, Saris DB, Creemers LB. Overexpression of hsa-miR-148a promotes cartilage production and inhibits cartilage degradation by osteoarthritic chondrocytes. Osteoarthritis Cartilage 2014;22:145-53.

47. Liu B, Qiang L, Wang GD, Duan Q, Liu J. LncRNA MALAT1 facilities high glucose induced endothelial to mesenchymal transition and fibrosis via targeting miR-145/ZEB2 axis. Eur Rev Med Pharmacol Sci 2019;23:3478-86.

48. Huang S, Zhang L, Song J, Wang Z, Huang X, Guo Z, et al. Long noncoding RNA MALAT1 mediates cardiac fibrosis in experimental postinfarct myocardium mice model. J Cell Physiol 2019;234: 2997-3006.

49. Tian W, Du Y, Ma Y, Gu L, Zhou J, Deng D. MALAT1-miR663a negative feedback loop in colon cancer cell functions through di- rect miRNA-lncRNA binding. Cell Death Dis 2018;9:857.

50. Tao F, Tian X, Ruan S, Shen M, Zhang Z. miR-211 sponges lncRNA MALAT1 to suppress tumor growth and progression through inhibiting PHF19 in ovarian carcinoma. FASEB J 2018;32:fj201800495RR.

51. Hong BK, You S, Yoo SA, Park D, Hwang D, Cho CS, et al. MicroRNA-143 and -145 modulate the phenotype of synovial fibroblasts in rheumatoid arthritis. Exp Mol Med 2017;49:e363.

52. Li S, Sun W, Zheng H, Tian F. Microrna-145 accelerates the inflammatory reaction through activation of NF- $\mathrm{KB}$ signaling in atherosclerosis cells and mice. Biomed Pharmacother 2018;103: 851-7.

53. Medvedeva EV, Grebenik EA, Gornostaeva SN, Telpuhov VI, Lychagin AV, Timashev PS, et al. Repair of damaged articular cartilage: current approaches and future directions. Int J Mol Sci 2018; 19:E2366.

54. Liang S, Gong X, Zhang G, Huang G, Lu Y, Li Y. The lncRNA XIST interacts with miR-140/miR-124/iASPP axis to promote pancreatic carcinoma growth. Oncotarget 2017;8:113701-18. 